\title{
Leiomioma parauretral de segmento proximal femenino: informe de un caso
}

\section{Female proximal segment paraurethral leiomyoma: case report}

José A. García-Muñiz ${ }^{1 *}$, César Cardona-Martínez², Jesús A. Beltrán-Marín², Arturo Luévano-González ${ }^{3}$, Omar Tapia-Gutiérrez ${ }^{4}$, Miriam Olivas-Villalobos ${ }^{2}$

${ }^{1}$ Departamento de Imagenología, Hospital Ángeles Chihuahua; ${ }^{2}$ Departamento de Imagenología, Hospital Ángeles Chihuahua; ${ }^{3}$ Departamento de Patología, Hospital Ángeles Chihuahua; ${ }^{4}$ Servicio de Urología, Hospital Ángeles Chihuahua. Chihuahua, México

\section{RESUMEN}

El leiomioma parauretral es una entidad patológica sumamente rara a nivel mundial. Aunque de causa no establecida, posee alta correlación con la exposición a estrógenos del músculo liso uretral, del cual deriva este tumor benigno. El diagnóstico por medio de imagenología es de alta complejidad. Se presenta el caso de una paciente femenina de 33 años nulípara, que acude a consulta para valoración por infección recurrente de vías urinarias; se realizó ultrasonografía (USG) transvaginal en la que se identificó una tumoración vesicouretral; se complementó el estudio con tomografía axial computarizada (TAC) y resonancia magnética nuclear (RMN); el resultado fue, como principal sospecha diagnóstica, leiomioma parauretral y luego se tomó biopsia que confirmó el diagnóstico.

Palabras clave: Leiomioma parauretral. Leiomioma uretral. Leiomiosarcoma. Adenomioma. Diagnóstico por imagenología.

\section{ABSTRACT}

The paraurethral leiomyoma is an extremely rare pathological entity worldwide, of not established etiology however it has a high correlation with estrogen exposure of the urethral smooth muscle from which this benign tumor is derived. The diagnosis through imaging is highly complex. In this case, we present a 33-year-old female nulliparae, who goes to the clinic for assessment for recurrent urinary tract infections, transvaginal and subprapubic USG is performed, where vesicourethral tumor is identified, it is complemented 
with CTscan and NMR, with the main suspicion being a paraurethral leiomyoma, Subsequently, a biopsy is taken and a diagnosis is confirmed.

Key words: Paraurethral leiomyoma. Urethral leiomyoma. Leiomyosarcoma. Adenomyoma. Imaging diagnosis.

\section{INTRODUCCIÓN}

Los leiomiomas son lesiones benignas compuestas de células de músculo liso y su presentación más frecuente ocurre en el tracto gastrointestinal y el sistema urogenital. En el sistema genitourinario se han documentado casos localizados en útero, pelvis renal, pared vesical y uretra en mujeres, casi siempre originados en el segmento proximal ${ }^{1,2}$.

Un leiomioma parauretral verdadero se define como una neoplasia mesenquimatosa benigna que crece en el espacio parauretral o el tabique vesicovaginal sin comunicación con uretra, vejiga o vagina, está encapsulada y no muestra infiltración a estructuras anexas ${ }^{1}$. La presentación clínica de estas lesiones es sumamente variable, su curso clínico es asintomático (50\%), con sensación de cuerpo extraño (78.6\%) y disuria (28.6\%). Los estudios de imagenología de elección para el diagnóstico y la diferenciación entre un leiomioma parauretral verdadero y uno uretral son el USG transvaginal y la RMN, respectivamente ${ }^{2}$.

El leiomioma parauretral femenino es una neoplasia extremadamente rara que se ha relacionado con la estimulación de los receptores del músculo liso por estrógenos de origen endógeno en edad reproductiva y durante el embarazo o de origen exógeno, en mujeres perimenopáusicas. Los diagnósticos diferenciales que se deben considerar son quiste de la glándula de Bartholin, quiste del conducto de Gardner, leiomiosarcoma, carcinoma uretral, carúncula uretral, divertículo uretral, prolapso de la mucosa uretral, quistes de la pared vaginal, endometriosis, pólipo fibroso uretral, absceso del conducto de Skenne, absceso, pólipo fibroepitelial, adenomioma y tumor mixoide ${ }^{3-6}$.

Los leiomiomas uretrales se diferencian de los parauretrales por la protrusión a través de la mucosa uretral y esto se puede valorar con estudios de imagenología o por cistoscopia; la disección quirúrgica tiene alto riesgo de lesión en la mucosa vesical; si la resección quirúrgica no presenta transgresión de la mucosa, su origen no se deriva de dicho sitio. Los leiomiomas uretrales tienden a ser fijos en comparación con los leiomiomas parauretrales, que a menudo no se fijan al resto de la pared uretral $^{2,7}$. Es estrictamente necesario el estudio histopatológico para el diagnóstico definitivo; el pronóstico es excelente, con escasas recidivas tumorales y no se han informado casos de malignización de la lesión primaria ${ }^{3}$. Desde el punto de vista macroscópico, la lesión es de consistencia firme, redondeada y sésil, con bordes lisos, coloración rosada o grisácea, típicamente de origen en segmento uretral proximal y pared posterior ${ }^{3,4}$.

La uretra se caracteriza en el plano histológico por una capa circular de músculo liso en 
el tercio proximal y dos capas musculares (músculo liso y estriado) en el tercio medio; el tercio distal se deja con una capa muscular lisa y otra externa estriada en forma de omega con abertura posterior; la composición muscular referida conforma un complejo esfintérico. Por esa disposición estructural y el origen de este tumor derivado de células de musculo liso, en la mayoría de los casos su desarrollo ocurre en el segmento uretral proximal y aún con menor frecuencia en el segmento uretral distal ${ }^{8,9}$.

La distinción entre leiomioma vaginal, uretral y parauretral es con frecuencia muy difícil debido a su posición anatómica cercana. En sentido anterior a posterior, hay cuatro capas que separan la pared vaginal exterior del espacio parauretral: el epitelio, la submucosa, las bandas fibromusculares que contienen músculo liso y la fascia periuretral ${ }^{1,8}$. Los leiomiomas parauretrales tienden a aparecer en las áreas entre el tabique vesicovaginal o el espacio parauretral ${ }^{10}$. Se presume que la rareza extrema del leiomioma parauretral se debe a una reducción drástica del tejido que se encuentra en el tabique vesicovaginal o el espacio parauretral ${ }^{11}$.

En la RMN, un leiomioma no degenerativo se describe como una lesión isointensa potenciada en T1 e hipointensa potenciada en T2 que se refuerza con el contraste; de manera característica se presenta en la forma de lesiones homogéneas, bien definidas con vascularización aumentada; los leiomiomas degenerativos tienen apariencias variables de mayor heterogeneidad con bordes menos definidos, pero no se cuenta con criterios radiológicos bien establecidos para definirlos ${ }^{4}$.

\section{CASO CLÍNICO}

Paciente femenina de 33 años de edad, originaria de Chihuahua, con antecedentes heredofamiliares sin relevancia para el caso, nulípara, con ciclos menstruales regulares, sin tratamiento de fecundidad o consumo de anticonceptivos; el último estudio de Papanicolaou realizado ocho meses antes resultó normal; no tiene operaciones previas. Entre los antecedentes personales patológicos se indica que se diagnosticó con síndrome de Barlow en 2015, el cual se trató con metoprolol durante un año. Cuenta con diagnóstico de hiperreactividad sistémica a múltiples alergenos ambientales (polen, huevo, plátano, pescado, tomate), por lo cual comenzó tratamiento con esquema de inmunoterapia un año y seis meses antes con aplicaciones subcutáneas, tres días por semana, complementado con cetirizina y montelukast.

Desde unos dos años antes ha cursado con infecciones de vías urinarias (IVU) recurrentes (cada dos a tres meses); se sometió a diferentes esquemas de antibioticoterapia por indicación de su ginecólogo; la última IVU se presentó un año antes. Se agrava su padecimiento tres meses antes del abordaje clínico, con síntomas caracterizados por dolor abdominal hipogástrico tipo cólico intermitente sin irradiación, disuria, pujo y tenesmo vesical, urgencia miccional e incontinencia urinaria a los cambios de posición, sobre todo en decúbito supino y dispareunia. En la exploración física no se encontraron alteraciones. Se realizó un ultrasonido transvaginal en el que se identificó útero en anteroversoflexión y lesión ovalada a nivel de cuello vesical que al parecer surge de la uretra en su porción anterior, de bordes definidos, 
heterogénea, sin calcificaciones y delimitada, sin aparente invasión a estructuras adyacentes, con vascularidad periférica y central aumentada (Fig. 1A); también se realizó una exploración con ultrasonido por vía suprapúbica en la que que confirmó lo hallado en la exploración con el transductor transvaginal (Fig. 1B). Con posterioridad se solicitó una urotomografía axial computarizada (uro-TAC) con contraste intravenoso que identificó una franca tumoración sólida redondeada de contornos lisos y bien definidos, retropúbica, extravesical, con dimensiones de $3.6 \times 3.2 \times 3.8 \mathrm{~cm}$ y densidad de 30 unidades Hounsfield (UH) en la fase simple (Fig. 2A), $63 \mathrm{UH}$ en fase arterial y 50 UH en fase tardía (Fig. 2B) sin áreas de necrosis central o periférica, proyectada en dirección posterosuperior hacia la pared vesical adyacente y ligeramente hacia abajo en dirección de la uretra, quizá dependiente de la pared anterior uretral; además, se realizó RMN con contraste intravenoso que delineó una lesión discretamente heterogénea retropúbica, hipointensa, potenciada en T1 (Fig. 3A) e hiperintensa y potenciada en T2 (Fig. 3B), con captación de medio de contraste, posterior al pubis y anterior a la uretra ovalada de contornos regulares y bien definidos. No tenía signos de infiltración a la mucosa uretral ni al piso vesical, en el que se observó un origen muy probable en el espacio parauretral de la pared anterior uretral a nivel del cuello vesical. La principal sospecha diagnóstica, con base en los estudios de imagenología, fue la de un leiomioma parauretral o bien leiomioma uretral, pero sin descartar otro origen menos probable, como leiomiosarcoma, adenomioma o pólipo fibroepitelial.

A continuación, en el abordaje quirúrgico por el servicio de urología, se obtuvo toma de

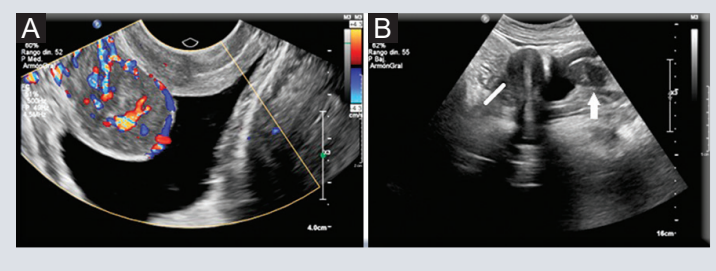

Figura 1. A: Ultrasonografía transvaginal con Doppler a color en corte sagital que delinea una tumoración de bordes definidos con vascularización aumentada. B: Ultrasonografía por vía suprapúbica en corte sagital que muestra una lesión tumoral sólida y heterogénea (flecha blanca) y útero en anteroversoflexión sin alteraciones (línea blanca).
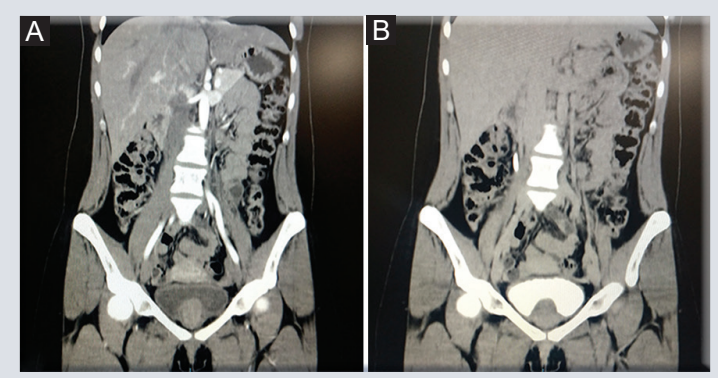

Figura 2. A: Uro-TAC en corte coronal con fase de contraste arterial que muestra una tumoración sólida isodensa redondeada de contornos lisos y bien definidos, con dimensiones de $3.6 \times 3.2$ x $3.8 \mathrm{~cm}$. B: Corte coronal en fase de contraste tardía.

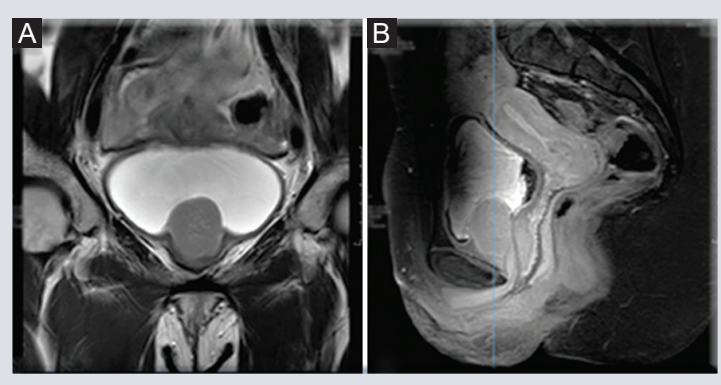

Figura 3. A: RMN con contraste intravenoso en fase tardía (corte coronal) potenciada en T1; se observa una lesión hipointensa de bordes bien definidos. B: Corte sagital en fase tardía potenciada en T2; se identifica una lesión hiperintensa dependiente de la pared anterior uretral con efecto de volumen hacia el trígono vesical y con origen presumible en la capa muscular externa. 
biopsia por medio de cistoscopia (cistoscopio rígido de Storz, camisa 21, lente 3 de $0^{\circ}$ ), en la que se encontraron como hallazgos uretra con compresión anterior extrínseca en la región proximal y segmento de cuello vesical lateral derecho con superficie lisa que también protruía hacia la vejiga, sin infiltrar la mucosa. Se efectuaron cortes longitudinales y se recogieron dos biopsias fusiformes de $1 \times 2 \mathrm{~cm}$, blanquecinas y homogéneas, que se enviaron a estudio transoperatorio que identificó histopatología consistente con leiomioma parauretral, por lo que se programó para resección completa como mejor opción terapéutica y curativa. En el procedimiento anterior al protocolo quirúrgico se practicó una incisión tipo Pfannenstiel y se diseccionó por planos hasta llegar al espacio de Retzius; se disecó y se colocó separador Alexis, se incidió la vejiga de modo transversal y se expuso la tumoración; luego se diseccionó la muscular vesicouretral para realizar la escisión completa de la tumoración (Fig. 4).

\section{Patología quirúrgica}

Se describe una lesión que corresponde a un nódulo parauretral: peso de $13 \mathrm{~g}$ y dimensiones de $4.5 \times 3 \mathrm{~cm}$. Se reconoció rodete de mucosa uretral vesical de $1 \times 0.8 \mathrm{~cm}$ con la porción central excavada y con cambio por cauterio secundario a toma de biopsia (Fig. 5A). Se aplicó tinta china, se realizan cortes longitudinales y se encontró la superficie de corte levemente arremolinada, de tono café claro con puntilleo hemorrágico (Fig. 5B). Los cortes histológicos teñidos con hematoxilina y eosina (H-E) muestran la lesión compuesta por haces de músculo liso orientados en diferentes direcciones. Las

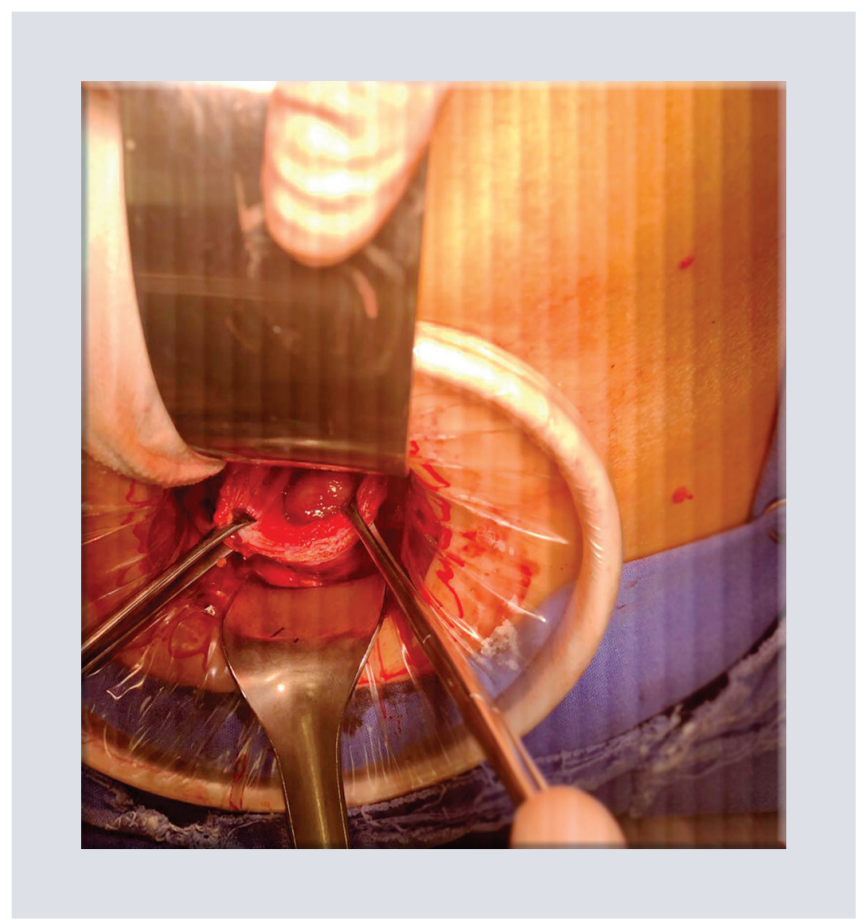

Figura 4. Exposición de la lesión tumoral en la escisión quirúrgica.

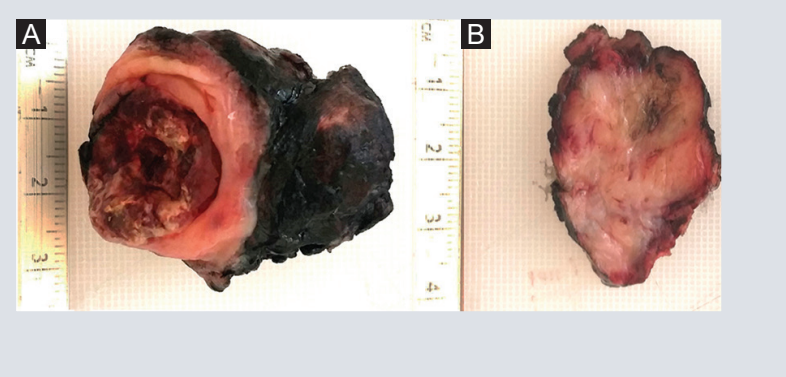

Figura 5. A: Pieza quirúrgica macroscópica correspondiente al leiomioma; resección de 4.5 × $3 \mathrm{~cm}$. B: Corte longitudinal de la pieza macroscópica.

células son fusiformes con núcleos alargados levemente hipercromáticos. Se observó leve variación en el tamaño. No se identificaron cambios degenerativos en relación con daño por cauterio ni necrosis fibrinoide. No hubo evidencia de atipia y los bordes laterales de mucosa vesical mostraron el epitelio sin alteraciones histológicas (Fig. 6). 


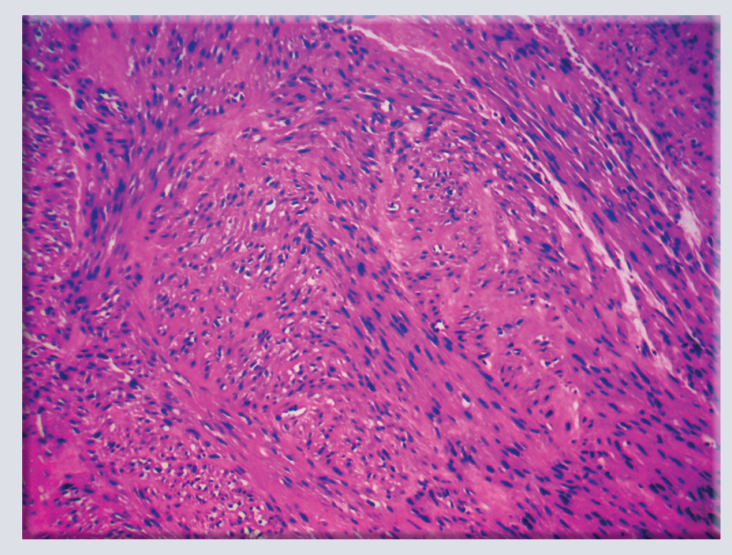

Figura 6. Corte histológico que corresponde al leiomioma con patrón fascicular de células musculares lisas sin atipia, con bordes de resección negativos para lesión y bordes laterales de mucosa uretral sin alteraciones (hematoxilina-eosina, aumento original s40x).

\section{ANÁLISIS Y DISCUSIÓN}

Los leiomiomas parauretrales tienen una incidencia mundial extremadamente baja, con una prevalencia de 1 en 50,000 muestras quirúrgicas. Aparecen con mayor frecuencia en la pared anterior vaginal y el tamaño más común es de $1 \mathrm{a} 5 \mathrm{~cm}^{12}$, que es el intervalo de tamaño en el que se encontró la lesión en este caso. El curso clínico de los leiomiomas parauretrales verdaderos es muy similar al de los uretrales, por lo que es sumamente difícil definir primero que se trata de un leiomioma y segundo su origen histológico real. Sin embargo, la integración interdisciplinaria de los departamentos de imagenología y patología, desde el punto de vista diagnóstico con el servicio de urología en la vertiente clínica y quirúrgica, ofrece suficiente evidencia para apoyar un diagnóstico de leiomioma parauretral verdadero, ya que reúne las características establecidas distintivas, es decir, una "neoplasia mesenquimatosa que crece en el espacio parauretral o en el tabique vesicovaginal sin comunicación con la uretra, vejiga o vagina"1. Si bien la etiología no se encuentra descrita del todo en las publicaciones médicas, sí existen factores de riesgo adjuntos (ya descritos) que incrementan la posibilidad de desarrollar un leiomioma parauretral; no obstante, la paciente no contaba con ninguno de ellos, lo cual le confiere mayor singularidad a este caso. Como ya se mencionó, la evolución biológica de estos tumores es benigna y su hallazgo suele ser incidental en una revisión de control, dado que el paciente refiere sensación de cuerpo extraño o IVU recurrentes. En este caso, la paciente ya presentaba un cuadro de larga evolución con IVU recurrentes que se relacionó con la exacerbación de otros síntomas genitourinarios tres meses antes de este abordaje clínico. En cuanto a su localización, este tipo de tumores uretrales y parauretrales aparece casi siempre en segmentos proximales en alrededor del $80 \%$ de los $\operatorname{casos}^{13}$; lo más común es que se origine de la pared posterior, pero en este caso el leiomioma era dependiente de la pared anterior. La mejor prueba diagnóstica y en la que se evidencia su origen por medio de estudios de imagen es la RMN con contraste IV potenciada, tanto en T1 como en T2 y analizado en sus diferentes cortes.

El abordaje quirúrgico depende de la localización del tumor. Cuando la lesión se encuentra en la uretra distal se recomienda escisión local; sin embargo, cuando la lesión se halla en la uretra proximal se puede realizar por abordaje transuretral ${ }^{3,14}$. En este caso, el abordaje para la resección se llevó a cabo suprapúbico, que también es una opción quirúrgica, y se extirpó la lesión en su totalidad con excelente evolución posoperatoria de la paciente sin complicaciones inmediatas o mediatas y sin recurrencia al seguimiento clínico. El análisis histopatológico 
mostró el diagnóstico definitivo y el patrón celular típico de un leiomioma con células musculares en disposición fascicular y alargamiento de núcleos sin datos de atipia.

\section{CONCLUSIONES}

El valor agregado y el tema central que representa este caso clínico es la baja incidencia mundial de este tipo de tumores, su difícil identificación y correcta diferenciación entre leiomioma uretral, que es más común, y un leiomioma parauretral verdadero; esta distinción se realizó por medio de los estudios de imagen complementarios, en especial el ultrasonido para identificar la lesión y la RMN para reconocer el origen real, además de descartar otros diagnósticos diferenciales, el principal de ellos un leiomiosarcoma. Con posterioridad se realizó un adecuado abordaje quirúrgico para la resección de la lesión y por último el departamento de patología del hospital definió el diagnóstico; no obstante, este tipo de entidades patológicas representa todavía un verdadero reto diagnóstico para el médico radiólogo y el equipo multidisciplinario que lo aborda por su extrema singularidad.

\section{CONFLICTO DE INTERESES}

Todos los autores declaran no tener ningún conflicto de intereses en la realización de este trabajo.

\section{RESPONSABILIDADES ÉTICAS}

Protección de personas y animales. Los autores declaran que para esta investigación no se han realizado experimentos en seres humanos ni en animales.

Confidencialidad de los datos. Los autores declaran que han seguido los protocolos de su centro de trabajo sobre la publicación de datos de pacientes.

\section{Derecho a la privacidad y consentimiento} informado. Los autores han obtenido el consentimiento informado de los pacientes o sujetos referidos en el artículo. Este documento obra en poder del autor de correspondencia.

\section{BIBLIOGRAFÍA}

1. Rezai S, LoBue S, Bahl N, Chadee A, Gottimukkala S, Fishman A, et al. A true paraurethral leiomyoma, a case report and review of literature. Obstretics \& Gynecology Int J. 2017;6(5):1-6.

2. Carral-Valdez RA, Arellano-Sánchez E, Lira-Dale A, Aguilar-Baradas J, Pérez-Carillo O, Martín Del Campo S. Leiomioma parauretral: reporte de un caso y revisión de literatura. Rev Mex Urol. 2008;68(6):351-353.

3. Rodríguez-Alonso A, Pérez-García D, Núñez-López A, González-Piñeiro A, Ojea-Calvo A, Rodríguez-Iglesias B, et al. Leiomioma de uretra femenina. Actas Urol Esp. 2000;23(9):753-756.

4. Chaudhari VV, Patel MK, Douek M, Raman SS. MR imaging and US of female urethral and periurethral disease. RadioGraphics. 2010;30:1857-1874.

5. García-Morúa A, Gutiérrez-García JD, Valdés Sepúlveda F, Jaime-Dávila R, Gómez-Guerra LS. Cáncer de uretra femenino. Rev Mex Urol. 2009;69(3):127-130.

6. Ávalos-Sánchez R, Solano-Moreno HA, García-Pérez V, González-Espinoza E, Sánchez-Villaseñor G, Domínguez-Vidal G, et al. Carcinoma urotelial del tracto urinario superior. Experiencia en el Hospital de Especialidades del Centro Médico Nacional Occidente. Col Mex Urol. 2007;22(1):10-17.

7. Bautista-Gómez E, Monzoy-Vázquez J, Morales-García V, GalvánEspinosa H, Flores-Romero AL. Miomas uretrales: reporte de dos casos y revisión de bibliografía. Ginecol Obstet Mex. 2011;79(6):386-388.

8. Velarde-Ramos L, Gómez-Illanes R. Tumores de la uretra en el adulto. Rev Chil Urol. 2013:78(2):38-47

9. Mundy AR, Glass J. En: Stranding S, editor. Gray's anatomy. $39^{\circ}$ ed., section 7, chapter 94. Bladder, prostate and urethra. Male Urethra. Elsevier-Churchill Livingstone, 2008.

10. Perugia G, Ciccariello M, Pirolli F, Chinazzi A, Teodonio S, Borgoni G, et al. Paraurethral leiomyoma. Urology. 2012;79(4):e51-e52.

11. Martin JH, Gerrard ER. Paraurethral leiomyoma: Does making the distinction make a difference? Infect Urol. 2001;14(2):1-4.

12. Méndez-Delgadillo LM, Velázquez-Castellanos PI, Godoy-Rodríguez N. Leiomioma parauretral en la postmenopausia reporte de un caso. Gac Med Bol. 2018;41(1):61-63.

13. Chodisetti S, Namburi RR, Boddepalli Y. Female urethral leiomyoma presenting with acute urinary retention-a rare case with unusual presentation. Indian J Surg. 2015;77(1):128-129.

14. Vallmanya-Llena FR, Rijo-Mora E, Hernández-Pozo H, Del CantoAguirre M, Lorente-Garín JA, Gelabert-Mas A. Leiomioma uretral. Actas Urol Esp. 2007;31(10):1196. 